\title{
Early functional outcome of intercondylar humerus fractures fixed with precontoured dual plating in inverted Y-Fashion
}

\author{
Moradiya N. ${ }^{1}$, Shah $\mathrm{N}^{2}$, Joshi P. ${ }^{3}$, Joshi P. ${ }^{4}$ \\ ${ }^{1}$ Dr. Niravkumar Moradiya, Assistant Professor, Department of Orthopaedics, SMIMER (Surat Municipal Institute of \\ Medical Education and Research), Surat, Gujarat, India, ${ }^{2}$ Dr. Neel Shah, Senior Resident, ${ }^{3}$ Dr. Parth Joshi, Junior \\ Resident, ${ }^{4}$ Dr. Poojan Joshi, Junior Resident; above three authors are affiliated with Department of Orthopaedics, \\ GMERS Medical College and General Hospital, Gotri, Vadodara, Gujarat, India.
}

Corresponding Author: Dr. Niravkumar Moradiya, Assistant Professor, Department of Orthopedics, SMIMER (Surat Municipal Institute of Medical Education and Research), Surat, Gujarat, India. Permanent Address: C-801, Eiffel Tower, L. H Road, Surat, Gujarat. E-mail: moradiyanirav@gmail.com

\begin{abstract}
Objective: In this study we report the functional outcome of patients with intercondylar distal humerus fractures treated by precontoured angular stable anatomical locking plates in inverted Y fashion, using a standard approach. Materials and Methods: A total number of 24 patients with $\mathrm{AO}$ type $\mathrm{C}$ closed intercondylar distal humerus fractures were operated with open reduction through an olecranon osteotomy approach and internal fixation using two plates in inverted-Y fashion. Patients were followed at 4 weeks, 6 weeks, 12 weeks and thereafter every 6 months. They were evaluated using the Mayo Elbow performance index and Riseborough and Radin Score. Results: There were 9 (37.5\%) men and 15 (62.5\%) women with mean age of 46.72 years.79.17\% of the cases were following fall and rest following Motor vehicle accident. AO type $\mathrm{C} 2$ fractures accounted for $45.83 \%$ of cases; type $\mathrm{C} 3$ fractures accounted for $33.33 \%$ of cases and type $\mathrm{C} 1$ accounted for $20.84 \%$. According the Riseborough and Radin criteria, the results were Good in $14(58.33 \%)$ of patients, Fair in $9(37.50 \%)$ and Poor in 1(4.17\%). According to MEPI, we had Excellent results in $5(20.83 \%)$, Good in $12(50 \%)$, Fair in $6(25 \%)$ and Poor in $1(4.17 \%)$ patients. The mean MEPI was $82 \pm 18$. Thus according to MEPI, we achieved excellent to good results in $\sim 70 \%$ of patients with $100 \%$ union rate and complications less than $13 \%$. Conclusion: Intercondylar fractures of humerus treated by Dual plating in inverted Y-fashionoffers a reliable and stable fixation permitting early mobilization and comparable functional outcome with good union rates.
\end{abstract}

Keywords: Intercondylar fractures; distal humerus fractures; Distal humerus; Dual plate; precontoured anatomical locking plates

\section{Introduction}

Fractures of the distal humerus are uncommon injuries, accounting for only $0.5-2 \%$ of all adult fracturesand continue to provide operative challenges to the surgeon [1-3]. Distal humerus fractures commonly occur through a fall or more significant force onto a flexed elbow, transmitting forces through the thin-walled olecranon/coronoid fossae, occasionally splitting down through the articular margin. Historically, nonoperative management (i.e., "bag of bones") was advocated as the best form of treatment because of a lack of adequate surgical techniques and implant-related issues. During the past several decades, operative management became widely accepted as the best treatment for these injuries,

Manuscript Received: $8^{\text {th }}$ May 2018

Reviewed: $18^{\text {th }}$ May 2018

Author Corrected: $24^{\text {th }}$ May 2018

Accepted for Publication: $28^{\text {th }}$ May 2018 despite the complications associated with operative treatment. Distal humerus fractures are broadly categorized into those with intra-articular extension and those without. Intra-articular fractures are generally more challenging, at times requiring an olecranon osteotomy and extensive dissection.

Difficulties exist when managing distal humeral fractures because of challenges in obtaining ananatomic reduction, related ulnar nerve issues, heterotopic ossification, comminution, osteopenia, nonunion, and the complex decision-making regarding whether to treat operatively with total elbow replacement or open reduction and internal fixation (ORIF). Success frequently depends on various factors, including quality of reduction, fracture type and severity, and patient 


\section{Original Research Article}

compliance with physical therapy and lifting restrictions.Distal humerus fractures occur infrequently, but they represent $30 \%$ of all elbow fractures [1]. Most fractures of the distal humerus (50-70\%) areintraarticular and generally related to a simple fall and osteoporosis $[3,4]$. Distal humeral fractures occur in a bimodal distribution, but women over the age of 65 are most commonly affected.

Younger patients who sustain intra-articular fractures generally are involved in high-energy trauma [5]. Recent research indicates that the overall incidence of distal humeral fracturesis $5-30 / 100,000$ and is increasing because of a more active older population witha longer lifespan [4,6,7]. Compared with hip fractures associated with osteoporosis, distal humerus fractures generally occur to a more active patient who has a high-level of autonomy, frequently living independently [3]. Because of its increasing frequency, distal humeral fracture mana-gement is also increasing. The goals for the orthopedic surgeon treating these injuries should be to maintain function, decrease pain, and provide a stable ulnohumeral and radiocapitellar joint. Complications associated with distal humerus are nonunion, malunion, decreased motion, and instability.

\section{Aims and Objectives}

The purpose of the present study was to evaluate the earlyclinical outcome of inverted Y-shaped doubleplating in 24 patients with intercondylar fractures of the distal humerus using the post-operative evaluation criteria by Riseborough and $\operatorname{Radin}^{8}$ and the Mayo Elbow Performance Index (MEPI) [9].

\section{Materials and Methods}

Place of study: The study concluded 24 patients admitted to the tertiary care hospital with intercondylar distal humerus fracture.

Type of study: The study was prospective type of study.

Sampling methods: The simple random sampling method was used in this study.

Sample collection: The sample collection period was from June 2015 to May 2017.

Inclusion Criteria: patients above 18 years of age with closed intra-articular distal humerus fractures

\section{Exclusion Criteria}

- patients less than 18 years of age

- fracture in a paralytic limb

- open fractures

- patients medically unfit for surgery

- inflammatory arthritis or other arthritis of the injured elbow

- Old neglected fractures

Primary \& Pre-operative Management: Upon arrival in the department of casualty, thorough clinical examination of patients was done including neurovascular examination. Radiography in form of $\mathrm{x}$ - rays, traction $\mathrm{x}$ - rays and CT scans with 3D reconstruction were done to know the exact geometry of fracture. Written informed consent was obtained from every patient regarding the surgery and inclusion in the study. The patients were evaluated using a standardized preanaesthetic work-up, and other associated injuries were treated using the appropriate treatment for that particular disease.

Surgical Management: Surgery was performed either under general anaesthesia or under brachial block with or without tourniquet control. The patients were treated with primary open reduction and internal fixation using precontoured anatomical dual plating in inverted Y-Fashion. Posterior approach (standard midline) with olecrenon osteotomy was used in lateral decubitus position with arm supported and forearm hanging in all patients. Before osteotomy, ulnar nerve was identified and dissected and olecrenon was drilled with $6.5 \mathrm{~mm} \mathrm{CC}$ screw drill bill. Osteotomy was done with oscillating saw and final osteotomy was done with osteotome.

The intercondylar articular surface was visualized and the articular surface was reconstructed anatomically (provisionally stabilized with ' $\mathrm{K}$ ' wires). The intraarticular reduction was stabilized with help of a $4.5 \mathrm{~mm}$ partially threaded $\mathrm{CC}$ screw passed over a guide wire. On attainment of a satisfactory articular reduction, the supracondylar ridges were examined and the shaft was reduced to the condyles and maintaining the medial and lateral ridges. The reduction was held with help of 


\section{Original Research Article}

bone holding forceps and an interfragmentary screw was used to attain reduction if required. Precontoured distal humerus $3.5 \mathrm{~mm}+2.7 \mathrm{~mm}$ locking plates were applied over the lateral and medial ridges in inverted Y-fashion. Length of both plates was kept unequal and less communited column was fixed first. Before definitive plate fixation, the elbow was placed through a range of motion to ensure there is no hardware impingement. Ulnar nerve was embedded in the soft tissue and a fat pad harvested locally was placed between the nerve and the medial plate if required. The olecranon osteotomy was stabilized with tension band wiring principle over long $6.5 \mathrm{CC}$ screw.

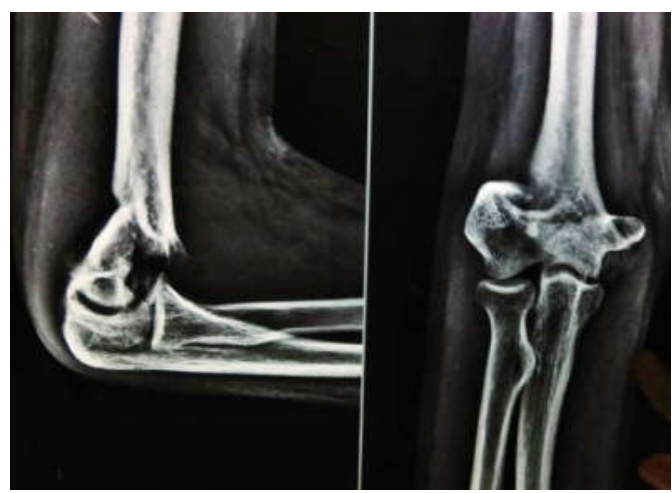

Pre-operative Radiograph

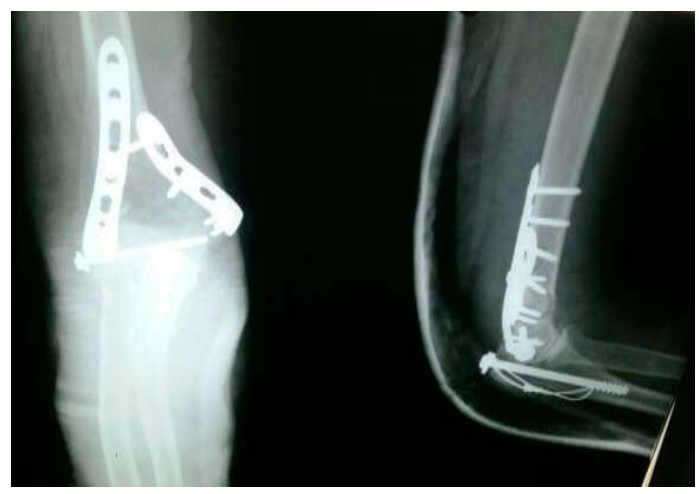

Post-operative Radiograph

Postoperative regime: Post-operatively, patients were instructed to move their fingers actively and limb elevation was maintained. Clean dressing was done on every third day post-operatively. On every dressing elbow was put to full range of motion once as per tolerability of the patients. Intravenous antibiotics were given for 6 days, later converted to oral until suture removal. Sutures were removed on the $14-16^{\text {th }}$ postoperative day and check x-ray in antero-posterior and lateral views were obtained. Patients were later discharged with the above elbow posterior POP slab and advised to perform active shoulder and finger movements. Patients were advised not to lift heavy weight or exert the affected upper limb.

Data collection, Follow-up \& Evaluation: At the end of 4 weeks, the plaster slab was then removed, an arm pouch was given and the patient was advised to do an active range of elbow movements as the pain permits. Patients were asked to return at 6 weeks, 12 weeks and thereafter every 6 months. The results were assessed at 3 months, 6 months and 1 year. At follow up, a detailed clinical examination was done and patients were assessed subjectively for the symptoms like pain, swelling and restriction of joint motion. Patients were instructed to perform physiotherapy in the form of active flexion-extension and pronation-supination without loading.

The functional assessment of the patient was done according to the Riseborough and Radin grading system and the Mayo Elbow Performance Index.

Stastical Method: All the statistical analysis was performed using Microsoft Excel Software. Data were presented as mean $\pm \mathrm{SD}$ and proportions as appropriate. Nonparametric data was compared using chi-square test while independent ttest was used to compare the parametric variables. A 2-sided p-value of less than 0.05 was considered statistically significant.

\section{Results}

The average age of the patients in our study was 46.72 years with a range of 18-72 years. 9 male patients and 15 female patients participated in the study; the female-to-male ratio was 1.67. The side involvement was almost equal with 11 fractures on the left side and 13 on the right. Majority of the patients 19 sustained injury due fall while 5 patients sustained injury due to road traffic accidents.

The fractures of the distal humerus were classified according to the AO classification. In our study maximum number (11 patients) had C2 type of fracture of the distal humerus followed by C3 type (8 patients). C1 type fracture was seen in 5 patients. We observed that complexity of fracture increased with increasing age. Patients with road traffic accidents had relatively complex fractures as compared to fall. 
Original Research Article

Maximum numbers of patients were operated within first week of injury. Delay in Surgery was either due to delayed presentation to the institution or due to management of comorbid conditions. One patient presented after one month of trauma. Mean duration between injury and surgery was $6.78 \pm 3.48$ days and mean duration of hospital stay was $8.84 \pm 3.37$ days. The average follow-up was 15.3 months with a range of 12-22 months.

Out of the 24 patients, 4 had hypertension, 2 had diabetes and 1 had both hypertension and diabetes which were treated pre-operatively according to physician's advice. 1 patient had distal radius fractures which were treated with percutaneous pinning and casting at the same time as the surgery for distal humerus. One patient had associated distal third femur fracture treated with Anatomical plating in the same sitting.

14 patients had range of motion of more than 100 degrees. 9 patients had range of motion between 80-100 degrees. Only 1 patient had range of motion less than 80 degrees. Mean range of motion was 117.083 degrees.

Table-1: Demographic distribution of patients.

\begin{tabular}{|c|c|c|}
\hline Variable & Numbers & $\%(n=24)$ \\
\hline \multicolumn{3}{|c|}{ Sex } \\
\hline male & 9 & 37.5 \\
\hline female & 15 & 62.5 \\
\hline \multicolumn{3}{|c|}{ Side Involved } \\
\hline Right & 13 & 54.16 \\
\hline Left & 11 & 45.84 \\
\hline \multicolumn{3}{|c|}{ AO types } \\
\hline C1 & 5 & 20.83 \\
\hline $\mathrm{C2}$ & 11 & 45.84 \\
\hline C3 & 8 & 33.33 \\
\hline \multicolumn{3}{|c|}{ Age (years) } \\
\hline 18-30 & 5 & 20.83 \\
\hline $31-40$ & 4 & 16.67 \\
\hline $41-50$ & 5 & 20.83 \\
\hline $51-60$ & 6 & 25 \\
\hline 61-70 & 4 & 16.67 \\
\hline total & 24 & 100 \\
\hline
\end{tabular}

The final results were classified according to the Riseborough and Radin criteria and the Mayo Elbow Performance Index (MEPI). In our study, according the Riseborough and Radin criteria, the results were Good in 14 patients, Fair in 9 patients and Poor in 1patient. According to MEPI, we had Excellent results in 5 patients, Good in 12 patients, Fair in 6 patients and Poor in 1patient. The mean MEPI was $82 \pm 18$. Thus according to MEPI, we achieved excellent to good results in $\sim 70 \%$ of patients. Most of the good results were seen in type $\mathrm{C} 1$ and $\mathrm{C} 2$ fractures while the poor result was seen in type $\mathrm{C} 3$ fracture.

The most common complication in our study was post-operative stiffness of the elbow, seen in 2 patients. The second complication was superficial infection seen in 1patients. Three patients had complains of itching at local site seen 4-5 months after surgery. None of our patient had deep infection or implant failure. None of our patient had Non-unioneither at distal humerus or at olecrenon osteotomy site.

Stiffness was treated with physiotherapy in the form of CPM and ROM exercises, but for some stiffness persisted and led to poor results according to the final score. The superficial infections were treated by IV antibiotics according to culture $\&$ sensitivity and patients recovered without any long term complications.

No statistical significant relationship was found while comparing various fracture subtypes with parameters like interval between injury and surgery, duration of hospital stay and post-operative complications. Statistically no difference was found between younger and older patients in form of range of motion, mayo elbow performance score or pain at final follow up. Prolonged immobilization for a period of (4-6 weeks) had relatively poorer outcome in terms of range of motion and mayo elbow performance score at final follow up. 
Original Research Article

Table-2: Results.

\begin{tabular}{|c|c|c|c|c|}
\hline Results & Fair & Poor & Total \\
\hline $\begin{array}{c}\text { RR criteria } \rightarrow \\
\downarrow\end{array}$ & & & & \\
\hline Excellent & 5 & & & $\mathbf{5}$ \\
\hline Good & 9 & 0 & 0 & $\mathbf{1 2}$ \\
\hline Fair & 0 & 3 & 0 & $\mathbf{6}$ \\
\hline Poor & 0 & 6 & 1 & $\mathbf{1}$ \\
\hline
\end{tabular}

\section{Discussion}

Orientation of plates: It is well known fact and published studies proved that Open reduction plus dual plating remains 'Gold Standard' for management of intercondylar distal humerus fractures. There is much debate going on and controversy still prevails regarding orientation of plates for treatment of these fractures.

Currently 3 techniques available for placement of dual platings:

1 perpendicular 90-90 plating recommended by AO group

2 Parallel plating recommended by O'driscoll [10]

3 Placement of plates in inverted Y-fashion

As far as perpendicular plating is concerned it was promoted early on by the AO group the biomechanical study of Helf et and Hotch kiss added credibility to this technique. A number of subsequent clinical studies revealed nearly $75-85 \%$ good to excellent results with 90-90 plating [11].

Along term follow-up study at a mean of 19 years after injury by Doornberg concluded that the long term results of open reduction and internal fixation of 19 Type $\mathrm{C}$ fractures of the distalpart of the humerus treated with perpendicular orientation aresimilar to those reported in the short term [13]. They suggested that the results are durable over time. The clinical experience with parallel plating has not been as extensive or with longer follow up, however current reports reveal no evidence of failure of the fixation and comparable clinical results as with $90-90$ plating.

Biomechanical evidence: Which technique is more stable? Several biomechanical studies compared parallel plating with perpendicular 90-90 orientation, concluding that parallel plating with additional use of bolts was favorable to perpendicular plating. Their observations were supported by Arnander who concluded that, parallel plating was superior to the perpendicular orientation although they expressed concern that placing aplate lateral can be technically difficult $[14,15]$. Kimball found that the risk of delayed union or nonunion increased by the extensive subperiosteal elevation with parallel plating orientation [16].

Jacobson concluded that perpendicular plate orientation was strongest in the sagittal plane while Korner stated that perpendicular plating had increased stiffness to torsional and anteroposterior bending forces [15,17]. Korner showed that locking plates have a substantial advantage in poor bone quality or if significant metaphysical comminution is present [17]. Otherwise they concluded that there was no difference in plate type and that plate position is critical. Schwartz found similar stabilization among both plate orientations. Wong tested both fixation methods and concluded that both methods may be above the threshold necessary for early motion and predictable fracture healing, rendering the marginal strength of parallel plating clinically unimportant [18-19].

R. K. Guptaet.al., concluded that Dorsal application of both the plates provides steady enough configuration, requiring less extensive dissection and ulnar nerve retraction thereby resulting in a low incidence of complications [20-21].

Our Observation: Although parallel plating much discussed and used more frequently being a new procedure, we still used dual plates in inverted $\mathrm{Y}$ fashion. Only reason was to avoid much dissection on both columns in sagittal plane which may compromise origin of common flexors and extensors of forearm from both epicondyles. It may increases chances of myositis ossificans post operatively. Furthermore the flat surface of plate is less likely to irritate the ulnar nerve as compared to the edge of the plate when placed medially over the supracondylar crest. We found 


\section{Original Research Article}

posterior placement of both plates was having sufficient strength to hold fragments together and being anatomical they maintain normal anatomical flexion of distal humerus.

According to our clinical experience, placement of 3.5 $\mathrm{mm}$ locking screws in distal fragment (as used previously and still today) are too large to be placed for Cancellous bone in distal humerus. Although $3.5 \mathrm{~mm}$ screws are must required for fixation of proximal fragment, $2.7 \mathrm{~mm}$ locking screws are better for distal fragment for two reasons: one for better purchase in small fragment and second was ease of placement in different direction (variable angle locking) in presence of $4.0 \mathrm{~mm}$ CC screw (which we used in all patients for intercondylar compression). It is proven and well known fact that one must never over compress $4.0 \mathrm{~mm}$ $\mathrm{CC}$ screw while achieving intercondylar compression to prevent trochlear stenosis. We followed the principle very well.

Regarding post-operative $\mathrm{x}$-ray, it does not always coincide with the functional result. Those with Excellent function of the elbow may demonstrate a distorted radiographic appearance, and vice versa. On final $\mathrm{x}$-ray, there may be nearly perfect anatomical restoration but poor functional capacity, usually due to joint stiffness [8]. Hence the surgeon may have to compromise appearance (both clinically and radio graphically) in function point of view [12].

Clinical Observations: The average age of patients in our study was 46.72 year with maximum population in the $5^{\text {th }}$ decade. This was comparable to study by Subramanian V et.al. where mean age was 40.83 years [22]. In our study, there were 9 males patients and 15 female patients contrary to studies by R. K. Gupta et.al, Subramanian V et.al, Amite Pankaj et.al, and Swagat Mahapatra et. al, where there was male dominant population almost reverse of our scenario [20-24]. This difference in sex distribution may be due higher incidence of house-hold accidents among female sin our region. Observations regarding fracture classification and side involvement were similar and comparable to studies by R. K. Guptaetal., Subramanian Vetal., Amite Pankaj et al., and Swagat Mahapatra et al [20-24].

The different mechanism of injury in our study was due to major female population in our study having history of fall by house-hold accidents. All the fractures in our study achieved bony union by six months. There was no delayed union or nonunion. Mostauthors including Lee SK et al., Leigey DF etal., and many others have similar observation in their studies [25-29]. The mean Mayo
Elbow performance score was $82 \pm 18$. This was comparable to dual Y-plate study by Swagat Mahapatra et al [24]. Two studies by Rebuzziet al., (mean MEPS94.17) and Sanjiv Kumar et al., (mean MEPS-96.32) where they used parallel plating had MEPS of greater than 90 following operative fixation [30-31]. This may be attributed to the lower averageage group of the study population in these studies. A further functional evaluation was also done using the Riseborough and Radin criteria [8]. The results were Good in 14 patients, Fair in 9 patients and Poor in 1patient. According to the original study, they obtained Good results in 10 $(35.7 \%)$, Fair in $10(35.7 \%)$ and Poor in $8(28.5 \%)$ patients. Our results were likely more favorable due to advances in fixation and operative techniques.

The post-operative complication rates vary from 6 to 44 percent in various studies. Our study hadminimal complications. The application of both plates on the dorsal aspect instead of on the ridges results in a stable fixation requiring lesser soft tissue dissection, minimal periosteal stripping and minimal ulnar nerve retraction with decreased operative time and lesser complications like deep infection. Gupta et al., derived asimilar conclusion from their study $[20,21]$.

Due to minimal soft tissue and periosteal stripping it was difficult to maintain reduction using reduction clamps. So it is advisable to provisional fix the fracture fragments with $\mathrm{K}$ wires for maintaining reduction and ease of plate application. Our study is not free of limitations which include smaller sample size, shorter follow-up and lack of comparison group. Long term follow-up for dorsal plating and clinical trials are needed to compare different plate orientation.

\section{Conclusion}

In conclusion, locking plate is a versatile implant providing stable-enough fixation and helping restoration of normal anatomy for good result and early rehabilitation. The results of our study do indicate that precontoured anatomical Dual plates in inverted Yfashion is a useful option in distal humerus fractures even type $\mathrm{C}$ injuries with communited small distal fragments which permits early mobilization and has a good functional outcome with minimal complications. This may in future become the preferred method of fixation by most surgeons.

What this study add to existing knowledge: Apart from parallel and perpendicular plating dual plating, posterior plating is equally effective and excellent method for treating distal humerus fracture. 
Contribution by Authors: Niravkumar Moradiya contributed to the study design, implementation of the research, verification of the analytical methods, supervised the findings of this work and to the writing of the manuscript. Niravkumar Moradiya and Neel Shah operated all the patients. Parth Joshi, Pujan Joshi and Neel Shah assessed follow up of patients and their functional outcome. Parth Joshi and Pujan Joshi collected data and analysed it. All authors discussed the results and contributed to the final manuscript.

Abbreviations: CC screw- Cannulated Cancellous screw; MEPI - Mayo Elbow Performance Index; CPM Continuous passive motion; ROM - range of motion.

Conflict of interest: None declared.

Funding: Nil, Permission from IRB: Yes

\section{References}

1. Zlotolow DA, Catalano LW 3rd, Barron OA, Glickel SZ. Surgical exposures of the humerus. J Am Acad Orthop Surg. 2006 Dec; 14(13):754-65.

2. Morrey BFFractures of the distal humerus: role of elbow replacement. Orthop Clin North Am. 2000 Jan; 31 (1): 145-54.

3 Charissoux, J.L., Vergnenegre, G., Pelissier, M., et al.. Epidemiology of distal humerus fractures in the elderly. Orthopaedics \& Traumatology: Surgery \& Research. Volume 99, Issue 7, November 2013, Pages 765-769 DOI: 10.1016/j.otsr.2013.08.002 (PMID: 24075780)

4. Robinson CM, Hill RM, Jacobs N, et al. Adult distal humeral metaphyseal fractures: epidemiology and results of treatment. J Orthop Trauma. 2003 Jan;17 (1): $38-47$.

5. Galano GJ, Ahmad CS, Levine WN. Current treatment strategies for bicolumnar distal humerus fractures. J Am Acad Orthop Surg. 2010 Jan;18(1): 20-30.

6. Palvanen M, Kannus P, Niemi S, Parkkari J. Secular trends in distal humeral fractures of elderly women: nationwide statistics in Finland between 1970 and 2007 DOI:10.1016/j.bone.2009.11.025.

7. Sheps D.M., Kyle kemp., Hilde brand Kevin. Population-based incidence of distal humeral fractures among adults in a Canadian urban center. Current Orthopaedic Practice 2011; 22(5):437-442 September 2011.DOI: 10.1097/BCO. 0b013e318229d0b5

\section{Original Research Article}

8. Riseborough EJ, Radin EL. Intercondylar T fractures of the humerus in the adult. A comparison of operative and non-operative treatment in twenty-nine cases. J Bone Joint Surg Am. 1969 Jan;51(1):130-41.

9. Longo UG, Franceschi F, Loppini M, et al. Maffulli N, Denaro V. Rating systems for evaluation of the elbow. DOI:10.1093/bmb/ldn023

10. O'Driscoll SW. Optimizing stability in distal humeral fracture fixation. 2005;14(1): S186-S194. DOI: $10.1016 /$ j.jse.2004.09.033

11. Helfet DL, Hotchkiss RN. Internal fixation of the distal humerus: a biomechanical comparison of methods. J Orthop Trauma. 1990;4(3):260-4.

12. Keon-Cohen BT. Fractures at the elbow. J Bone Joint Surg Am. 1966 Dec;48(8):1623-39.

13. Doornberg JN, van Duijn PJ, Linzel D, et al. Surgical treatment of intra-articular fractures of the distal part of the humerus. Functional outcome after twelve to thirty years. DOI:10.2106/JBJS.F.00369

14. Arnander MW, Reeves A, MacLeod IA, et al. A biomechanical comparison of plate configuration in distal humerus fractures. doi: 10.1097/BOT.0b013e 31816 edbce.

15. Jacobson $\mathrm{SR}^{1}$, Glisson RR, Ur baniak JR. Comparison of distal humerus fracture fixation: a biomechanical study. J South Orthop Assoc. 1997 Winter; 6(4):241-9.

16. Kimball JP, Glowczewskie F, Wright TW. Intraosseous blood supply to the distal humerus. DOI:10.1016/j.jhsa.2007.02.019

17. Korner J, Lill H, Muller LP. 2003. The LCPconcept in the operative treatment of distal humerus fractures-biological, biomechanical and surgical aspects. Int J Care Injured 34: S-B20-S-B30. PMID: 14580983

18. Schwartz A, Oka R, Odell T, Mahar A. Biomechanical comparison of two different periarticular plating systems for stabilization of complex distal humerus fractures. DOI:10.1016/j.clinbiomech.2006. 04.018

19. Wong AS, Baratz ME. Elbow fractures: distal humerus. J Hand Surg Am. 2009 Jan;34(1):176-90. doi: 10.1016/j.jhsa.2008.10.023. 
20. Rakesh Kumar Gupta, Vinay Gupta, Dickey Richard Marak. Locking plates in distal humerus fractures: study of 43Patients. Chinese Journal of Traumatology Volume 16, Issue 4, August 2013, Pages 207-211.https: //doi.org/10.3760/cma.j.issn.1008-1275.2013.04. 004

21. Gupta R, Khanchandani P. Intercondylar fractures of the distal humerus in adults: a critical analysis of 55 cases.Injury. 2002 Jul;33(6):511-5.

22. Subramanian V, Rajanish R Menon, Kumaran CM. Functional outcome of supracondylar - inter condylar fractures of humerus in adults treated by $\mathrm{Y}$ plate fixation. Kerala Journal Of Orthopaedics Volume 27| Issue 1 | January 2014

23. Amite Pankaj, G Mallinath, Rajesh Malhotra, et al., Surgical management of intercondylar fractures of the humerus using triceps reflecting anconeus pedicle (TRAP) approach. Indian journal of orthopaedics. Indian J Orthop. 2007 Jul-Sep; 41(3): 219-223. doi:10. 4103/0019-5413.33686 PMID: 21139748

24. Swagat Mahapatra, Vineet Thomas Abraham. Functional Results of Intercondylar Fractures of the Humerus Fixed with Dual Y-Plate; A Technical Note. Bull Emerg Trauma. 2017 Jan; 5(1): 36-41. PMID: 28246622

25. Lee SK, Kim KJ, Park KH, Choy WS. A comparison between orthogonal and parallel plating methods for distal humerus fractures: a prospective randomized trial DOI:10.1007/s00590-013-1286-y.

\section{Original Research Article}

26. Lan X, Zhang LH, Tao S, et al. Zhang Q, Liang $\mathrm{XD}$, Yuan BT, Xu WP, Yin P, Tang PF. Comparative study of perpendicular versus parallel double plating methods for type $\mathrm{C}$ distal humeral fractures. Chin Med J (Engl). 2013 Jun;126(12):2337-42

27. Aslam N, Willett K. Functional outcome following internal fixation of intraarticular [13] fractures of the distal humerus (AO type C). Acta Orthop Belg. 2004;70 (2):118- 22 .

28. Tian D, Jing J, Qian J, et al., Comparison of two different double-plate fixation [14] methods with olecranon osteotomy for intercondylar fracture of distal humerus of young adults. Exp Ther Med. 2013; 6(1): 147-151. doi: 10.3892/etm.2013.1102

29. Leigey DF, Farrell DJ, Siska PA, Tarkin IS. Bicolumnar 90-90 plating of low-energy distal humeral fractures in the elderly patient. DOI:10.1177/215145 8514526882

30. Rebuzzi E, Vascellari A, SchiavettiS.The use of parallel pre-contoured plates in the treatment of A and $\mathrm{C}$ fractures of the distal humerus. DOI:10.1007/s12306009-0051-1

31. Sanjiv Kumar, Sudhir Singh, Dharmenderkumar, et al., Intercondylar Humerus Fracture- Parallel Plating and Its Results. J Clin Diagn Res. 2015 Jan; 9 (1): RC01- RC04. doi: 10. 7860/ JCDR /2014 / 12137. 5479 .

\section{How to cite this article?}

Moradiya N, Shah N, Joshi P, Joshi P. Early functional outcome of intercondylar humerus fractures fixed with precontoured dual plating in inverted Y-Fashion. Surgical Update: Int J surg Orthopedics. 2018;4(2):75-82. doi:10.17511/ijoso.2018.i02.03. 